\title{
Influencia de los valores de los coeficientes de eficacia entre equipos de waterpolo ganadores y perdedores en el Campeonato de Europa de 1997 Influence the values of the coefficients of efficiency of waterpolo team winners and losers in the Championship of Europe 1997
}

\author{
Francisco M. Argudo Iturriaga, Encarnación Ruiz Lara, J. Arturo Abraldes Valeiras \\ Universidad Católica San Antonio de Murcia (España)
}

\begin{abstract}
Resumen: El presente estudio pretende alcanzar dos objetivos: el primero, averiguar los valores de los coeficientes de eficacia en los marcos situacionales, y el segundo, examinar la relación entre esos valores y la condición de ganador y perdedor en equipos masculinos y femeninos. Para la parte empírica se grabaron en vídeo la totalidad de los partidos de waterpolo disputados en el Campeonato de Europa celebrado en Sevilla durante el mes de Agosto de 1997, seleccionando cuarenta para el presente estudio. Tras la observación de los partidos elegidos, se valoró mediante el uso de coeficientes la eficacia en cada marco situacional. Se revelaron diferencias entre equipos ganadores y perdedores femeninos en los siguientes coeficientes: eficacia ofensiva en igualdad numérica (.022) y eficacia defensiva en igualdad numérica (.050); tomando como referencia un valor de $\mathrm{p}<.05$. Para concluir, decir que en ninguno de los doce coeficientes de eficacia, en waterpolo masculino, existen diferencias significativas entre equipos ganadores y perdedores. Mientras que en dos de los doce coeficientes de eficacia, en waterpolo femenino, existen diferencias significativas entre equipos ganadores y perdedores.
\end{abstract}

Palabras clave: waterpolo, eficacia, ganador, perdedor.

Abstract: TThe present study has intended to reach two objectives: the first one, to ascertain the values of efficacy in the micro situations of play, and the second, to examine the relation among the values of efficacy in male and female teams with the condition of winner or loser. Were recorded in video the totality of the matches of water polo disputed in the European Championship celebrated in Seville during the month of August of 1997, selecting forty for the present study. After the observation of the match selected, the use of coefficients valued itself by means of the efficacy in each situational framework. Significant differences among female winner and loser teams were revealed in the following coefficients: offensive efficacy in numerical equality (.022) and defensive efficacy in numerical equality (.050); taking as reference a value of $p<.05$. To conclude, to say that in none of the twelve coefficients of efficacy, in male water polo teams, exist significant differences among winner and loser. While in two of the twelve coefficients of efficacy, in female water polo teams, exist significant differences among winner and loser.

Key words: water polo, efficacy, winner, loser.

\section{Introducción}

Para toda persona que se ha preocupado en realizar una evaluación de la táctica en deportes colectivos, la primera dificultad con la que se ha encontrado ha sido la de poder establecer los parámetros que de verdad sean determinantes. La mayoría, por no decir la totalidad de las estadísticas sobre el rendimiento en deportes de equipo, no evalúan ciertamente la táctica. Por lo tanto, el problema es la especificación de los parámetros a fijarse en el ámbito de la táctica. No podemos cuestionar que resulte ilegítimo el planteamiento de ajustarse exclusivamente al proceso de ejecución, tal y como ha estado haciéndose hasta no hace mucho. Ni tampoco como señalan diversos autores, en el momento de la toma de decisión, por muy esencial que resulte de cara a la consecución final.

Se puede confrontar la existencia de trabajos muy profundos sobre el tema que nos ocupa. Tal es el caso de Lasierra y Escudero (1993), que definen lo que consideran la universalidad de las conductas fundamentales para cada jugador, en cada una de las situaciones que se le plantean en el juego (roles y subroles).

Para realizar la evaluación táctica en el alto rendimiento deportivo (ARD), según Sánchez (1996), existen dos tipos de métodos:

1. La evaluación del aprendizaje táctico:

1.1. Concreción del nivel de asimilación de los contenidos tácticos.

1.2. Relacionada siempre con la actuación individual.

1.3. Estarían perfectamente indicados los métodos de evaluación, del estilo de la Escala descriptiva que propone Lasierra (1993).

2. La evaluación táctica en el ARD:

2.1. Concreción del grado de asimilación del sistema táctico.

2.2. Eficacia de nuestro juego colectivo.

2.3. Detección de errores en la asimilación de los contenidos tácticos.

Fecha de recepción: 05-04-07 - Fecha de aceptación: 28-04-07

Correspondencia: Francisco Argudo Iturriaga

Campus de los Jerónimos, s/n. 30107

GUADALUPE (Murcia) ESPAÑA

E-mail: AAbraldes@pdi.ucam.edu
2.4. Relacionada con la actuación individual y colectiva.

2.5. Habrá que utilizar métodos que relacionen el sistema táctico diseñado, con la actuación real tanto individual como colectiva, así como la eficacia de esta actuación real.

El mismo autor comenta: «lo que nos debe importar en el $A R D$, es conocer el grado de eficacia del sistema de juego realizado (eficacia real de nuestro juego colectivo) y su afinidad con el diseñado anteriormente (relación entre el sistema de juego diseñado y la actuación real del equipo). Obviamente, esto no debe excluir el estudiar otros parámetros que también resultan interesantes para analizar la actuación de nuestro equipo Desde este prisma es a partir del cual debemos organizar nuestro sistema de observación».

Sin embargo no coincidimos con este autor, los métodos que propone no son tales, más bien se debería referir a ellos como a dos situaciones, la primera relacionada con el aprendizaje táctico en etapas de formación o el aprendizaje de un nuevo sistema de juego (no queda claro cual de las dos detalla) y la segunda enlazada con el alto rendimiento deportivo.

En deportes colectivos podemos distinguir tres tipos de evaluación, según Sánchez (1996):

1. Momentánea: la que se realiza en plena competición.

2. Corto plazo: se efectúa después de cada partido.

3. Medio o largo plazo: se realiza después de un ciclo de trabajo determinado (una fase en la liga, tras la temporada, etc.).

De todas las definiciones existentes sobre la evaluación podemos extraer una conclusión: «es la valoración del rendimiento de algo». En el deporte podemos evaluar la preparación física, la preparación técnica, la preparación psicológica y la preparación táctica. Esta evaluación se podrá realizar, según Gayoso (1983), fuera de la situación de juego (in vitro) o en la situación de juego (in vivo). El mismo autor afirma que los dos tipos de evaluaciones tienen ventajas y unos inconvenientes:

1. La evaluación in vitro permite una estandarización más rigurosa de la performance a evaluar, facilita la comparación del jugador consigo mismo observando un periodo precedente, o la comparación de un jugador con otros jugadores, y asegura sin duda alguna, una fidelidad de la medida. 
2. De forma genérica, la administración de un test in vitro es más fácil que la evaluación del jugador in vivo.

3. La evaluación in vivo permite medir elementos que escapan al modo de evaluación in vitro, ello es debido a los elementos tácticos y a los elementos de ética que intervienen en la competición (in vivo).

4. A pesar de las tentativas que son hechas para validar las medidas in vitro, éstas siguen siendo menos válidas que las medidas in vivo, sobre todo en las valoraciones de las habilidades deportivas. Las medidas in vivo son $100 \%$ válidas.

\begin{tabular}{|c|c|c|}
\hline \multicolumn{3}{|c|}{ VALIDEZ Y FIDELIDAD DE LAS MEDIDAS IN VITRO Y DE LAS } \\
MEDIDAS IN VIVO
\end{tabular}

Continúa diciendo: «el comportamiento táctico del deportista, según actúe en un tipo de deporte $u$ otro, estará condicionada a los aspectos tácticos, lo que nosotros denominaremos variables tácticas. Sabemos que no todas las modalidades deportivas tienen el mismo grado de exigencias en el comportamiento táctico, ya que difieren de si son deportes individuales, colectivos o de lucha. Lógicamente las tareas de evaluación de los comportamientos tácticos de estos grupos de deportes cobrará una mayor o menor importancia. En los deportes individuales será menor y en los deportes colectivos será mayor».

Para nosotros el proceso de la evaluación táctica (ver figura 2) se inicia con la cuantificación de las variables tácticas de los sistemas de juego. ¿Con qué se mide?, con la hoja de registro (ver figura 3). El siguiente paso es la valoración de la eficacia de esos sistemas de juego. ¿Con qué se mide?, con los coeficientes de eficacia. ¿Y qué se obtiene?, los valores de eficacia.

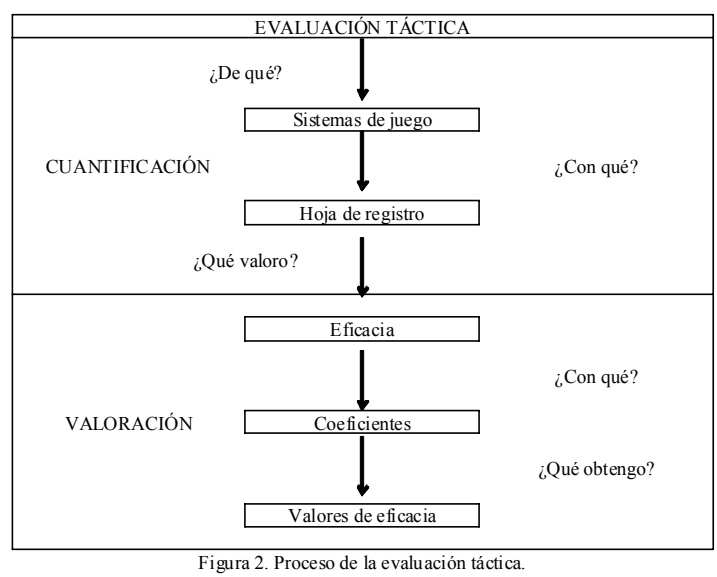

Gayoso (1983), considera que los resultados obtenidos en las evaluaciones de las situaciones de juego nos darán unas constantes que podremos denominarlas rendimientos o eficacia, términos que en realidad son similares. La eficacia, según el mismo autor, podemos considerarla como el resultado de las acciones correctamente ejecutadas dentro de una cantidad de intentos o ensayos:

Eficacia $=\mathrm{N}^{o}$ de acciones correctas $\mathrm{x} 100 / \mathrm{N}^{o}$ total de acciones realizadas.

El mismo, determina que dentro de la eficacia podremos distinguir dos tipos:

1. La eficacia relativa o rendimientos primarios/relativos.

2. La eficacia absoluta o rendimientos absolutos.
Prosigue afirmando que: «esas dos clases de eficacia pueden ser aplicables a las acciones ofensivas. Dentro de ellas podemos considerar una eficacia relativa de las acciones ofensivas (o defensivas) y una eficacia absoluta de las acciones ofensivas (o defensivas). Pueden ser aplicadas a conceptos tales como tiros, pases, acciones de ataque y defensa, etc. Dentro del contexto general del ataque podemos encontrar las fórmulas que nos den la eficacia relativa y la eficacia absoluta. Si quisiéramos medir un aspecto especifico del ataque como pudiera ser el tiro, lo lograríamos de la siguiente forma:

1. Eficacia relativa $=N^{o}$ detiros a porteríax $100 / N^{o} d e$ tiros ensayados.

2. Eficacia absoluta $=N^{\circ}$ de goles conseguidos $x$ 100/ $N^{\circ}$ de tiros ensayados.

Dentro del contexto general de la defensa, también podemos aplicar la medición de los rendimientos o eficacia, tanto relativos como absolutos aplicando las mismas fórmulas anteriores. El nivel de juego puede ser medido en los deportes colectivos con los consecuentes beneficios para conocer el nivel que un colectivo puede alcanzar en una competición. Considera que relacionando el tiempo de duración del encuentro con los puntos logrados y los logrados por el equipo adversario, se puede valorar el nivel de juego alcanzado, resultando el tiempo empleado en cada punto que decide la victoria o la derrota. Esta sería la fórmula:

Nivel de juego $=$ Tiempo real de juego (en minutos) $/ N^{o}$ de puntos a favor - $N^{o}$ de puntos en contra.

Reflexionando sobre todas las aportaciones de este autor, consideramos y estamos de acuerdo en su propuesta sobre la eficacia, la eficacia relativa y la eficacia absoluta. Pero en nuestra opinión comete dos errores, no diferenciar los marcos situacionales que se pueden presentar en cada deporte y el cálculo del nivel de juego. En éste incluye la variable tiempo y el marcador. El primero puede tener su relevancia, pero el segundo se anularía caso de empate y como resultado, si uno de los dos equipos gana, determinaría un valor numérico de dificultosa interpretación y validez, al no contar con una tabla de baremación. Por último, y ahora sí coincidiendo plenamente con este autor, consideramos que las mediciones y evaluaciones de los comportamientos tanto «in vivo» como «in vitro», son de capital importancia.

Los objetivos de este trabajo fueron: a) hallar los valores de los coeficientes de eficacia en cada marco situacional yb) analizar la relación entre esos valores y la condición de ganador y perdedor. La hipótesis de este trabajo fue que existen diferencias en los valores de eficacia entre los equipos ganadores y perdedores en ambos sexos.

\section{Método}

\subsection{Población}

La población estudiada pertenece al Campeonato de Europa de Sevilla 1997, es decir, fueron objeto de estudio todos los equipos nacionales que participaron en el mismo, por lo que se les supone un nivel de

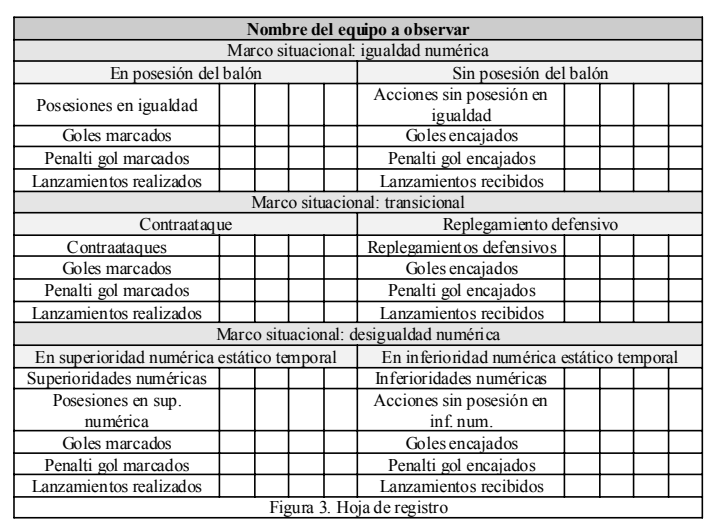


homogeneidad. Los partidos disputados en este Campeonato fueron 88 y los analizados para el presente estudio 40.

\subsection{Instrumentos}

Todos los partidos han sido analizados con una hoja de registro (Argudo, 2000), instrumento desarrollado para la evaluación táctica cuantitativa en waterpolo (ver figura 3).

Quedando conceptualizada de la siguiente forma:

1. Igualdad numérica: situación de juego en la que están presentes, en el campo de juego, todos los componentes de ambos equipos que pueden coincidir en la piscina al mismo tiempo según el reglamento (seis jugadores y un portero por equipo).

1.1. Posesiones en igualdad numérica: número de veces que un equipo puede, si lo desea, atacar (35").

1.2. Goles marcados: número de veces que el balón entra en la portería contraria de forma reglamentaria.

1.3. Penalti gol marcados: tras señalar un pena máxima se consigue un gol de forma reglamentaria.

1.4. Lanzamientos realizados: cantidad de modalidades biomecánicas de ejecución, que en presencia del reglamento, se llevan a cabo contra la portería contraria con intención de conseguir un gol.

1.5. Acciones sin posesión en igualdad numérica: número de veces que un equipo puede, si lo desea, defender (35").

1.6. Goles encajados: número de veces que el balón entra en la propia portería de forma reglamentaria.

1.7. Penalti gol encajados: tras señalar un pena máxima no se consigue un gol.

1.8. Lanzamientos recibidos: cantidad de modalidades biomecánicas de ejecución, que en presencia del reglamento, se llevan a cabo contra la propia portería con intención de conseguir un gol.

2. En transición: situación de juego con superioridad o inferioridad numérica dinámica temporal, contraataque o replegamiento defensivo respectivamente.

2.1. Contraataque: es una situación de juego, tras recuperar la posesión del balón, en la cual el equipo se encuentra en superioridad numérica dinámica temporal.

2.2. Replegamiento defensivo: es una situación de juego, tras la pérdida de la posesión del balón, en la cual el equipo se encuentra en inferioridad numérica dinámica temporal.

3. Desigualdad numérica: situación de juego en la que un equipo queda con un jugador más, o menos, por exclusión temporal de un jugador del equipo contrario (20") ó por el resto del partido si es excluido sin sustitución.

3.1. Superioridad numérica estático temporal: situación de juego en la que un equipo queda con un jugador más por exclusión temporal de un jugador del equipo contrario (20").

3.2. Posesiones en superioridad numérica estático temporal: número de veces que un equipo puede, si lo desea, atacar en ventaja numérica con respecto al rival.

3.3. Inferioridad numérica estático temporal: situación de juego en la que un equipo queda con un jugador menos por exclusión temporal de un jugador del equipo contrario (20").

3.4. Sin posesión en inferioridad numérica estático temporal: número de veces que un equipo puede, si lo desea, defender en desventaja numérica con respecto al rival.

Las variables objeto de estudio han sido la condición de ganador y perdedor al terminar el partido y los valores de eficacia obtenidos a partir de los coeficientes propuestos por Argudo (2000) que se desarrollan a continuación:

1. Coeficiente de eficacia ofensiva en igualdad numérica (CEOIN):

$\mathrm{CEOIN}=\left(\mathrm{N}^{\circ}\right.$ goles marcados $+\mathrm{N}^{\circ}$ penalti gol marcados $) \times 100 / \mathrm{N}^{\circ}$ posesiones. (CEOIN $>+$ ).

2. Coeficiente de resolución ofensiva en igualdad numérica (CROIN):

$\mathrm{CROIN}=\left(\mathrm{N}^{\circ}\right.$ goles marcados $+\mathrm{N}^{\circ}$ penalti gol marcados $) \times 100 / \mathrm{N}^{\circ}$ lanzamientos realizados. (CROIN $>+$ ).
3. Coeficiente de eficacia defensiva en igualdad numérica (CEDIN):

$\mathrm{CEDIN}=\left(\mathrm{N}^{\circ}\right.$ goles encajados $+\mathrm{N}^{\circ}$ penalti gol encajados $) \times 100 / \mathrm{N}^{\circ}$ acciones sin posesión. $(\mathrm{CEDIN}<+)$.

4. Coeficiente de resolución defensiva en igualdad numérica (CRDIN):

$\mathrm{CRDIN}=\left(\mathrm{N}^{\circ}\right.$ goles encajados $+\mathrm{N}^{\circ}$ penalti gol encajados $) \times 100 / \mathrm{N}^{\circ}$ lanzamientos recibidos. $(\mathrm{CRDIN}<+)$.

5. Coeficiente de eficacia ofensiva del contraataque (CEOC):

$\mathrm{CEOC}=\left(\mathrm{N}^{\circ}\right.$ goles marcados $+\mathrm{N}^{\circ}$ penalti gol marcados $) \times 100 / \mathrm{N}^{\circ}$ contraataques. (CEOC $>+$ ).

6. Coeficiente de resolución ofensiva del contraataque (CROC):

$\mathrm{CROC}=\left(\mathrm{N}^{\circ}\right.$ goles marcados $+\mathrm{N}^{\circ}$ penalti gol marcados $) \times 100 / \mathrm{N}^{\circ}$ lanzamientos realizados. (CROC $>+$ ).

7. Coeficiente de eficacia defensiva del replegamiento defensivo (CEDRD):

$\mathrm{CEDRD}=\left(\mathrm{N}^{\mathrm{o}}\right.$ goles encajados $+\mathrm{N}^{\circ}$ penalti gol encajados $) \times 100 / \mathrm{N}^{\circ}$ replegamientos defensivos. (CEDRD $<+$ ).

8. Coeficiente de resolución defensiva del replegamiento defensivo (CRDRD):

$C R D R D=\left(N^{o}\right.$ goles encajados $+N^{o}$ penalti gol encajados $) \times 100 / N^{\circ}$ lanzamientos recibidos. $(\mathrm{CRDRD}<+)$.

9. Coeficiente de eficacia ofensiva en superioridad numérica estática temporal (CEOSNET):

CEOSNET $=\left(\mathrm{N}^{\circ}\right.$ goles marcados $+\mathrm{N}^{\circ}$ penalti gol marcados $) \times 100 / \mathrm{N}^{\circ}$ posesiones. (CEOSNET $>+$ ).

10. Coeficiente de resolución ofensiva en superioridad numérica estática temporal (CROSNET):

CROSNET $=\left(\mathrm{N}^{\circ}\right.$ goles marcados $+\mathrm{N}^{\circ}$ penalti gol marcados $) \times 100 / \mathrm{N}^{\circ}$ lanzamientos realizados. (CROSNET $>+$ ).

11. Coeficiente de eficacia defensiva en inferioridad numérica estática temporal (CEDINET):

CEDINET $=\left(\mathrm{N}^{\circ}\right.$ goles encajados $+\mathrm{N}^{\circ}$ penalti gol encajados $) \times 100 / \mathrm{N}^{\circ}$ acciones sin posesión. (CEDINET $<+$ ).

12. Coeficiente de resolución defensiva en inferioridad numérica estática temporal (CRDINET):

CRDINET $=\left(\mathrm{N}^{\circ}\right.$ goles encajados $+\mathrm{N}^{\circ}$ penalti gol encajados $) \times 100 / \mathrm{N}^{\circ}$ lanzamientos recibidos. (CRDINET $<+$ ).

\subsection{Procedimiento}

El método de filmación partía del enfoque inicial al centro del terreno de juego, para una vez que uno de los dos equipos entraba en posesión del balón realizar una técnica de barrido centrando la imagen en el medio campo donde se desarrollaba la acción de juego. La observación de los partidos se hizo de forma consensuada entre cuatro especialistas entrenados (Anguera et al., 2000; Anguera, 2003).

\subsection{Análisis estadístico}

Las pruebas de homogeneidad de varianza se calcularon a través del estadístico de Levene. Posteriormente se realizó una ANOVA de un solo factor seguida por la prueba de Tukey para el análisis de las diferen-

\begin{tabular}{cc}
$\begin{array}{c}\text { Tabla 1. Valores de significancia de los valores de eficacia } \\
\text { entre equipos masculinos ganadores y perdedores }\end{array}$ \\
\hline Hombres ganadores - perdedores \\
\hline CEOIN & $\mathrm{p}>0.129$ \\
CROIN & $\mathrm{p}>0.742$ \\
CEDIN & $\mathrm{p}>0.129$ \\
CRDIN & $\mathrm{p}>0.742$ \\
CEOC & $\mathrm{p}>0.735$ \\
CROC & $\mathrm{p}>0.487$ \\
CEDRD & $\mathrm{p}>0.735$ \\
CRDRD & $\mathrm{p}>0.437$ \\
CEOSNET & $\mathrm{p}>0.433$ \\
CROSNET & $\mathrm{p}>0.765$ \\
CEDINET & $\mathrm{p}>0.433$ \\
CRDINET & $\mathrm{p}>0.765$ \\
\hline
\end{tabular}


cias estadísticamente significativas entre los valores de eficacia en cada coeficiente y la condición de ganador o perdedor al finalizar el partido. Todo el tratamiento estadístico mencionado se realizó con el paquete estadístico SPSS, aceptándose un nivel de confianza del 95\% y una probabilidad de error del 5\% (nivel de significación de .05).

\section{Resultados}

La comparación entre los coeficientes de eficacia obtenidos en waterpolo masculino en los marcos situacionales, tras el análisis estadístico, ha proporcionado los siguientes resultados, tal y como se presentan en la Tabla 1.

Estos resultados extraídos muestran que los equipos masculinos no presentan diferencias significativas, entre ganadores y perdedores, en los coeficientes de eficacia.

La comparación entre los coeficientes de eficacia obtenidos en waterpolo femenino en los marcos situacionales, tras el análisis estadístico, ha proporcionado los siguientes resultados, tal y como se presentan en la Tabla 2.

Estos resultados extraídos muestran que los equipos femeninos presentan diferencias significativas en el coeficiente de eficacia ofensiva en igualdad numérica $(\mathrm{p}<.008)$ y en el coeficiente de eficacia defensiva en igualdad numérica $(\mathrm{p}<.050)$

\begin{tabular}{cc}
$\begin{array}{c}\text { Tabla 2. Valores de significancia de los valores de } \\
\text { eficacia entre equipos femeninos ganadores y perdedores. }\end{array}$ \\
\hline Mujeres ganadoras - perdedoras \\
\hline CEOIN & $\mathrm{p}<0.022^{*}$ \\
CROIN & $\mathrm{p}>0.281$ \\
CEDIN & $\mathrm{p}<0.050^{*}$ \\
CRDIN & $\mathrm{p}>0.551$ \\
CEOC & $\mathrm{p}>0.762$ \\
CROC & $\mathrm{p}>0.484$ \\
CEDRD & $\mathrm{p}>0.762$ \\
CRDRD & $\mathrm{p}>0.223$ \\
CEOSNET & $\mathrm{p}>0.444$ \\
CROSNET & $\mathrm{p}>0.993$ \\
CEDINET & $\mathrm{p}>0.444$ \\
CRDINET & $\mathrm{p}>0.993$
\end{tabular}

\section{Conclusiones}

Como conclusión principal de la evaluación táctica realizada en el Campeonato de Europa de 1997, se puede inferir que en ningún coeficiente de eficacia en categoría masculina y diez en categoría femenina existen diferencias significativas entre los equipos ganadores y perdedores. Por lo que la hipótesis planteada de la existencia de diferencias en los coeficientes de eficacia entre ganadores y perdedores, en categoría masculina, no se cumple. Mientras que en categoría femenina sólo se cumple en el coeficiente de eficacia ofensiva en igualdad numérica (CEOIN) y el coeficiente de eficacia defensiva en igualdad numérica(CEDIN).

\section{Referencias bibliográficas}

Anguera, M. T., etal. (2000). La metodología observacional en el deporte: conceptos básicos. Lecturas: Educación Física y Deportes, Revista Digital, 24. Extraído en octubre 5, 2002 disponible en: http://www.efdeportes.com.

Anguera, M. T. (2003). Diseños observacionales en la actividad fisica y el deporte: estructura, alcance, y nuevas perspectivas. Ponencia presentada en el II Congreso Mundial de Ciencias de la Actividad Física y del Deporte, Granada, España.

Argudo, F. (2000). Modelo de evaluación táctica en deportes de oposición con colaboración. Estudio práxico del waterpolo. Tesis Doctoral. Universidad de Valencia. Valencia.

Gayoso, F.(1983). Fundamentos de táctica deportiva. Madrid: Gayoso, $\mathrm{F}$.

Lasierra, G. (1993). Análisis de la interacción motriz en los deportes de equipo. Aplicación de los Universales Ludomotores al Balonmano. En «Apunts Educació física i Esports».

Lasierra, G y Escudero, P. (1993). Aproximación a la evaluación en los deportes de cooperación-oposición: a la búsqueda de sus aspectos distintivos. En «Apunts Educació física i Esports», Núm. 31, 86105.

Sánchez, F.(1996). Deportes de equipo: análisis funcional evaluación y aprendizaje de la táctica. Apuntes módulo 1.3.3. Master Universitario en Alto Rendimiento Deportivo. C.O.E.-U.A.M. Madrid: C.O.E.S. 